\title{
Accumulation Ratio Ctrough
}

National Cancer Institute

\section{Source}

National Cancer Institute. Accumulation Ratio Ctrough. NCI Thesaurus. Code C102426.

The trough concentration at steady state divided by the trough concentration during the initial dosing interval. 